\title{
Risk of Second Solid Malignancy among 1000 Kidney Transplanted Patients: A Single Center Study
}

\author{
Michele L. Santangelo*, Sergio Spiezia, Nicola Carlomagno, Concetta Dodaro, Cristina La Tessa, \\ Davide De Rosa, Maria Pina Piantadosi, Andrea Renda \\ Department of Surgery, Anesthesia and Emergency Science, School of Medicine, University of Naples “Federico II”, Naples, Italy \\ Email: *michele.santangelo@unina.it
}

Received October 4, 2012; revised November 4, 2012; accepted November 11, 2012

\begin{abstract}
In this study we focus on kidney-transplanted patients in order to investigate the correlation between immunodeficiency (ID) induced by immunosuppressive treatments and the incidence of solid multiple primary malignancies (MPM). Material of Study: From 1980 to 2010 we followed up 1000 kidney-transplanted patients (637M, 363F). This group was homogeneous for age, immunology (e.g. miss-match index), number of rejection events and for immunosuppressive therapy. Results: Out of 1000 kidney-transplanted patients we observed 70 patients (53M, 17F) with cancer disease and in 22 of them a multiple cancer has been found. Most of multiple cancers were synchronous and the association between cancer and rejection episodes were not significant. Discussion: In general population 1 out of 9 patients with a cancer would develop a second neoplasia during the course of his life, so, it would be logical to conclude that, from a merely theoretical and statistical point of view, long term transplanted patients potentially have a higher risk to develop MPM. But our series and literature review did not confirm it, probably because these patients die before the appearance of a second primary malignancy. Conclusions: Despite many observations regarding different types of tumors/precancerous lesions and their increased incidence in ID patients and despite the fact that immune suppression is a predisposing factor for the multicancer syndrome, at least theoretically, nowadays there are no significant statistical data favouring a correlation between ID and MPM in kidney transplanted patients.
\end{abstract}

Keywords: Multiple Primary Malignancies; Immunosuppressive Therapy; Kidney Transplantation

\section{Introduction}

The relationship between cancer onset and immune response has started to rouse great interest in the early 1900s, leading Ehrlich [1] to establish the conceptual basis of the immunological surveillance. The immune response is the result of complex interactions between various mechanisms that build the defensive system of the organism. The defects in one or more of these systems, induced by immunosuppressive drugs like in transplanted patients, compromise the immune response with consequent development of a secondary immune deficiency (ID) which can facilitate neoplastic onset.

After Billroth's first report in $19^{\text {th }}$ century, only in 1932 was reported a classification concerning 1259 patients with multiple primary malignancies (MPM). MPM were defined the tumours arisen in patients with the following clinical and histological characteristics: 1) malignant tumours from the histo-patologic point of view; 2) topographic distinct without connection with submucosal or intra-epithelial alterations (skip metastasis); 3) leaving

"Corresponding author. out a second tumour being not a metastasis of the first. In 1961 MPM were divided in simultaneous, synchronous and metachronous, when the diagnosis of the first tumour and a second one was contemporary, or there was less or more time than 6 months before the rising of the secondary one. The incidence of MPM is now increasing and this will be the trend in next years [2], so in this paper we analyzed our experience about secondary immunodeficiency (transplanted patients) and MPM.

\section{Methods and Material}

Through the analysis of medical records collected in our Department, we retrospectively examined 1000 kidney transplanted patients (630 male and 370 female) followed at Federico II University-Medical School between 1980-2010 [3,4]. The median follow-up was 10 years and the average graft survival 8 years. The group of patients was homogenous for age (range of age: 18 - 65), donor/ recipient immunology (e.g. miss-match index), number of rejection events and immunosuppressive therapy (1980-1984: corticosteroids [the oldest immunosuppression drug used], azathioprine [an old antiproliferative 
agents now usually considered as a "third agent" in triple immunosuppressive regimens]; 1984-1998 corticosteroids, calcineurin inhibitors [the most immunosuppressive drug used in maintenance therapy in the last two decades. Cyclosporine and tacrolimus are the most used calcineurin inhibitors and primarily suppress the activation of $\mathrm{T}$ lymphocytes by inhibiting the production of cytokines, specifically IL-2], basiliximab [an IL-2 receptor antagonist generally used as induction therapy], azathioprine; 1998-2010 corticosteroids, calcineurin inhibitors, basiliximab, mycophenolate [an new antiproliferative agents that interferes with DNA replication, producing cytostatic effects on $\mathrm{T}$ and $\mathrm{B}$ cells and generally used as a "third agent" in triple immunosuppressive regimens], mammalian target of rapamycin inhibitors [called also m-TOR inhibitors and generally identified with sirolimus and everolimus, represent an alternative to the long term calcineurin inhibitor-based regimen and its side effects]). We never used thymoglobuline drugs.

\section{Results}

Among 1000 kidney transplanted patients, 70 patients (7\%), 53 males (5.3\%) and 17 females (1.7\%), presented a cancer. 48 patients $(4.8 \%$ of all series and $68.5 \%$ of all cancer patients) developed a single cancer: 17 patients a skin single cancer (included melanoma) and 31 patients a no skin single cancer, representing respectively $1.7 \%$ and $3.1 \%$ of all series and $24.3 \%$ and $44.3 \%$ of all cancer patients. Instead 22 patients $(2.2 \%$ of all series and $31.5 \%$ of all cancer patients) presented multiple cancers: multiple skin cancer (included melanoma) 19 and solid MPM 3, representing respectively $1.9 \%$ and $0.3 \%$ of all series and $27.1 \%$ and $4.3 \%$ of all cancer patients. In detail, in the latter group, we observed the following associations: prostate/kidney cancer (synchronous, treated surgically, disease-free for 2 years); Kaposi's sarcoma/ MALToma (metachronous, medical and surgical treatment, disease-free for 1 year, exitus at 18 months) and lung cancer/squamous skin carcinoma (metachronous, medical and surgical treatment, disease-free at 8 months, exitus at 14 months). Multiple cancer associations and their onset time are detailed in Figures 1 and 2. Data about association between cancer and rejection episodes are not significant: rejection episodes were found only in 4 patients and just 1 of them developed a second cancer.

\section{Discussion}

Today the high standards in surgical and anaesthesiaological-resuscitation procedures and clinical management of subjects undergoing transplant allow to obtain extremely positive results on short and medium-term survival of both organs and patients [5-10]. Incidence and aggressiveness of neoplastic diseases appear to be

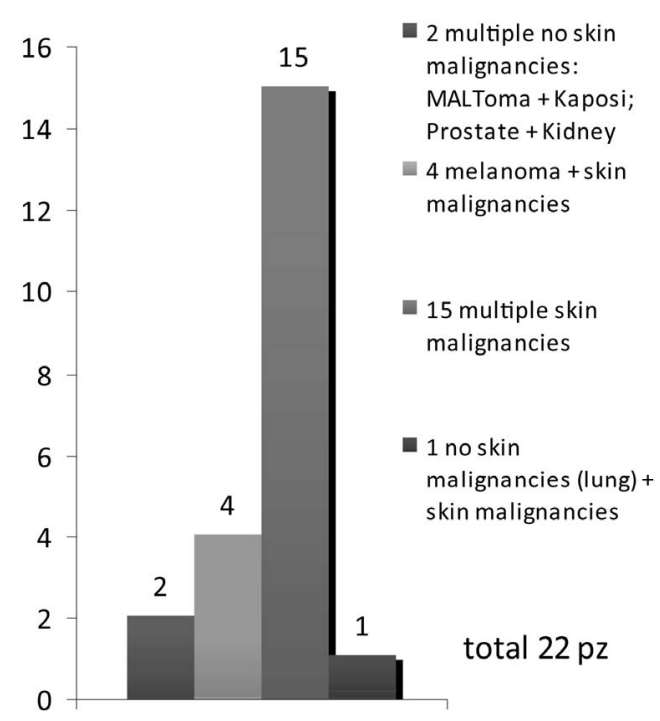

Figure 1. Cancers associations among 1000 kidney transplant patients.

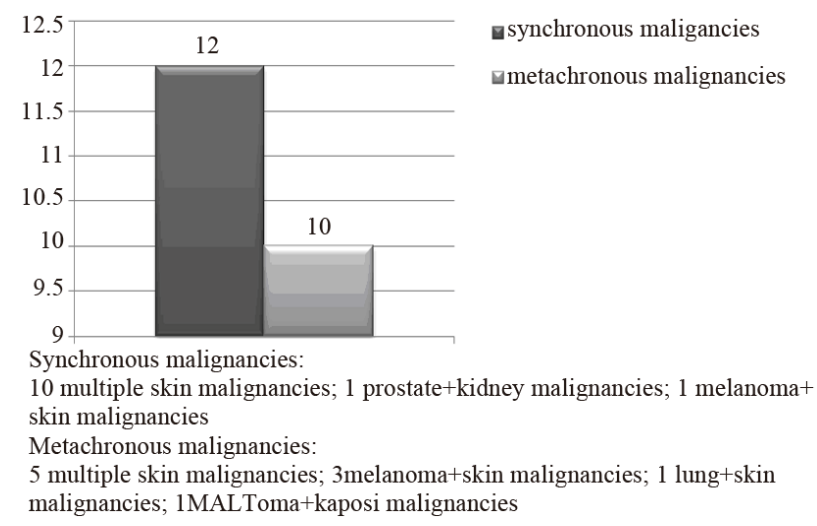

Figure 2. Onset time of MPM among 1000 kidney transplant patients.

remarkably increased in this group of patients compared to the general population of corresponding age. It has been calculated that the prevalence of a neoplastic disease at 10 years post-renal transplant is from $20 \%$ to $30 \%$, with peaks of $40 \%$ at 20 years [11-15]. The association between pharmacological immune suppression and increased risk of cancer is a very widely discussed topic in clinical medicine and attention has been given to different aspects of the matter, such as duration of immune suppression therapy, intensity of the treatment and used drugs [16]. It has been calculated that if malignant tumors carried a lower mortality rate and were more uniformly distributed in the general population, we could still expect to find that 1 out of 9 cancer patients would develop a second neoplasia during the course of their life and that within this group 1 out of 27 will probably have a third primitive cancer [15-21]. This statistical projection obviously refers to the general population. Therefore, it would be logical to conclude that from a merely theo- 
retical and statistical point of view immuno-suppressed patients have a potentially higher risk to develop MPM [15,18,22-24]. However our experience and literature review don't seem to support this conclusion. First of all it may be due to patients' cancer related death occurring before the appearance of a "second primary malignancy". Furthermore it is very hard to find transplant patients, who survive from a first cancer and continue immune suppressive treatment long enough to develop a second primary cancer related to his iatrogenic immune deficiency state. Therefore transplanted subject undergoing immune suppressive treatment can have three different ways to develop a second cancer: 1) Subjects recovered from a cancer and undergoing a transplant showing a new cancer at follow-up; 2) Subjects recovered from a cancer undergoing transplant and developing a new cancer transmitted by the donor; 3) Subjects developing Multiple Primary Cancer after transplant.

In our study we have experienced only class of patients developing MPM after transplant. We did not observe patients included in group A because we have followed-up only 8 patients in this condition. None of the patients included in group B was observed probably because this is a purely theoretical condition.

\section{Conclusion}

Despite many observations regarding different types of tumors/precancerous lesions and their increased incidence in ID patients and despite the fact that immune suppression is surely a predisposing factor for the multicancer syndrome, at least theoretically, there are no significant statistical data favouring a correlation between ID and MPM. We may therefore assume that it is hard to see a second cancer developing in ID patients because of their shorter life expectancy. Nevertheless, from the few reports found in literature and from our experiences, we can only conclude that MPM in immune suppressed patients generally: are synchronous, regard the same organ or tissue but situated in different sites, have a viral etiology and at least one lesion is easily explorable (e.g. skin cancer) such to allow an early diagnosis and treatment. It is our opinion that the treatment of MPM in immune suppressed patients should be as intensive as possible, so to obtain a complete eradication of the lesion. Moreover, it might be useful to eventually suspend the immune suppression treatment or switch to other drugs; this therapeutic approach has given so far good results in the treatment of same neoplastic diseases. In conclusion, the set of available clinical and epidemiological data allow to consider ID state as a risk factor for a neoplastic disease, but it does not furnish sufficient convincing evidence that an ID can facilitate the onset of an MPM. So, even if MPM do not seem to be a real problem today, they may become an important issue in the future, when new forms of treatment and stricter follow-ups will assure longer life expectancy in immune suppressed patients that have already had a cancer. Therefore, in potentially immune suppressed patients (e.g. kidney transplant candidates), a great relevance must be given: 1) to preventive measures against oncoviral infections (e.g. vaccinations programme as it is already done for HBV and now starting for HPV); 2) to implementation of procedures for reducing the exposure to environmental oncogenetic factors (e.g. drugs, cigarette smoking, alcohol, sun exposure, etc. as it is already recommended to our patients); and 3) to strictly follow-up programs even on those apparatus/ organs (e.g. genitourinary system, skin, thyroid, liver, blood, bones) at higher cancer risk in these kind of patients.

\section{REFERENCES}

[1] A. Piro, A. Tagarelli, G. Tagarelli, P. Lagonia and A. Quattrone, "Paul Ehrlich: The Nobel Prize in Physiology or Medicine 1908,” International Reviews of Immunology, Vol. 27, No. 1-2, 2008, pp. 1-17. doi:10.1080/08830180701848995

[2] A. Renda and N. Carlomagno, "Nosography in: A. Renda (Ed): Multiple Primary Malignancies,” Springer Press, Milan, 2009, pp. 1-6.

[3] A. Picascia, P. Giannattasio, V. Grimaldi, et al., "Preliminary Analysis of Kidney Transplantation in Naples from 2000 to 2006," Tissue Antigens, Vol. 79, No. 6, 2012, p. 502.

[4] V. Grimaldi, A. Picascia, P. Giannattasio, et al., "Kidney Transplantation and Immunohematological Profile in Naples: A Coordinating Regional Experience from 2000 to 2006,” Vox Sanguinis, Vol. 103, No. 1, 2012, p. 269.

[5] J. Chinen, D. Anmuth, A. R. Franklin and W. T. Shearer, "Long-Term Follow-Up of Patients with Primary Immunodeficiencies," Journal of Allergy and Clinical Immunology, Vol. 120, No. 4, 2007, pp. 795-797. doi:10.1016/j.jaci.2007.07.044

[6] P. De Rosa, M. Santangelo, A. Ferrara, et al., "Suboptimal Kidney: The Experience of a Single Transplant Unit,” Transplantation Proceedings, Vol. 36, No. 3, 2004, pp. 488-490. doi:10.1016/j.transproceed.2004.02.008

[7] M. Santangelo, P. De Rosa, S. Spiezia, et al., "Healing of Surgical Incision in Kidney Transplantation: A Single Transplant Center's Experience," Transplantation Proceedings, Vol. 38, No. 4, 2006, pp. 1044-1046. doi:10.1016/j.transproceed.2006.03.005

[8] P. De Rosa, M. Santangelo, A. Scala, et al., "Difficult Vascular Conditions in Kidney Transplantation,” Transplantation Proceedings, Vol. 38, No. 4, 2006, pp. 10401043. doi:10.1016/j.transproceed.2006.03.073

[9] M. Santangelo, M. Zuccaro, P. De Rosa, et al., “Older Kidneys Donor Transplantation: Five Years’ Experience without Biopsy and Using Clinical Laboratory and Macroscopic Anatomy Evaluation,” Transplantation Pro- 
ceedings, Vol. 39, No. 6, 2007, pp. 1835-1837. doi:10.1016/j.transproceed.2007.05.039

[10] M. Santangelo, M. Clemente, S. Spiezia, et al., "Wound Complications after Kidney Transplantation in Nondiabetic Patients," Transplantation Proceedings, Vol. 41, No. 4, 2009, pp. 1221-1223. doi:10.1016/j.transproceed.2009.03.098

[11] IARC, "Epstein-Barr Virus and Kaposi's Sarcoma Herpesvirus/Human Herpesvirus 8," In: IARC Monographs on the Evaluation of Carcinogenic Risks to Humans, World Health Origination International Agency for Research on Cancer Press, Lyon, 1997, p. 70.

[12] EBPG Expert Group on Renal Transplantation, "European Best Practice Guidelines for Renal Transplantation, Section IV: Long-Term Management of the Transplant Recipient," Nephrology Dialysis Transplantation, Vol. 17, No. 4, 2002, pp. 1-67. doi:10.1093/ndt/17.suppl_4.1-a

[13] A. G. Sheil, "Organ Transplantation and Malignancy: Inevitable Linkage,” Transplantation Proceeding, Vol. 34, No. 6, 2002, pp. 2436-2437. doi:10.1016/S0041-1345(02)03169-X

[14] A. Gutierrez-Dalmau and J. M. Campistol, "Immunosuppressive Therapy and Malignancy in Organ Transplant Recipients: A Systematic Review,” Drugs, Vol. 67, No. 8, 2007, pp. 1167-1198. doi:10.2165/00003495-200767080-00006

[15] M. Santangelo, S. Spiezia, M. Clemente, et al., "Immunodeficiency and Multiple Primary Malignancies in: A. Renda (Ed): Multiple Primary Malignancies,” Springer Press, Milan, 2009, pp. 83-96.

[16] P. O’Donovan, C. M. Perrett, X. Zhang, B. Montaner, et al., "Azathioprine and UVA Light Generate Mutagenic Oxidative DNA Damage,” Science, Vol. 309, No. 5742, 2005, pp. 1871-1874. doi:10.1126/science.1114233

[17] S. R. Rheingold, A. I. Neugut and A. T. Meadows, "Secondary Cancer: Incidence, Risk Factors and Management,” In: E. Frey, Ed., Holland-Frei Cancer Medicine,
BC Decker Press, Hamilton, 2000.

[18] E. Strocchi, R. V. Iaffaioli, G. Facchini, et al., "StopFlow Technique for Loco-Regional Delivery of High Dose Chemotherapy in the Treatment of Advanced Pelvic Cancers," European Journal of Surgical Oncology, Vol. 30, No. 6, 2004. pp. 663-670. doi:10.1016/j.ejso.2004.04.005

[19] P. Piscicelli, A. Santoriello, F. M. Buonaguro, et al., "Incidence of Breast Cancer in Italy: Mastectomies and Quadrantectomies Performed between 2000 and 2005," Journal of Experimental and Clinical Cancer Research, Vol. 28, No. 1, 2009, p. 86. doi:10.1186/1756-9966-28-86

[20] L. Insabato, M. Siano, A. Somma, et al., "Extrapleural Solitary Fibrous Tumor: A Clinicopathologic Study of 19 Cases,” International Journal of Surgical Pathology, Vol. 17, No. 3, 2009, pp. 250-254. doi:10.1177/1066896909333779

[21] A. Tortoriello, G. Facchini, F. Caponigro, et al., "Phase I/II Study of Paclitaxel and Vinorelbine in Metastatic Breast Cancer,” Breast Cancer Research and Treatment, Vol. 47, No. 1, 1998, pp. 91-97. doi:10.1023/A:1005848921895

[22] H. M. Kauffman, W. S. Cherick, M. A. McBride, Y. Cheng and D. W. Hanto, "Deceased Donors with a Past History of Malignancy: An Organ Procurement and Transplantation Net Work/United Network for Organ Sharing Update,” Transplantation, Vol. 84, No. 2, 2007, pp. 272-274. doi:10.1097/01.tp.0000267919.93425.fb

[23] S. Maurea, M. Fusari, M. Imbriaco, et al., "Pitfalls in Diagnostic Imaging of Cystic Pancreatic Masses: A Case of True Cystic Lesion Mimicking a Mucinous Cystadenoma," Journal of the Pancreas, Vol. 13, No. 1, 2012, pp. 83-86.

[24] J. Dantal and E. Pohanka, "Malignancies in Renal Transplantation: An Unmet Medical Need,” Nephrology Dialysis Transplantation, Vol. 22, No. 1, 2007, pp. 4-10. doi:10.1093/ndt/gfm085 